\title{
HOMMAGE AU PROFESSEUR HENRI JAMMET
}

Lorsque j'ai lancé il y a près d'un an, l'idée de pụblier dans chaque numéro de la revue Radioprotection la "lettre de la présidente", je ne savais pas que j'allais un jour si proche consacrer l'une d'entre elles à la mémoire du professeur Henri Jammet. Sans doute parce qu'il était toujours aussi actif et présent auprès de nous tous. Sans doute aussi parce qu'il portait en lui une volonté permanente de renouvellement, de créativité.

Il fut avant tout un pionnier, et les textes qui ont été rassemblés par la rédaction de la revue sous la direction de Henri Métivier en témoignent.

Il était en même temps une vedette des médias. Avant de l'avoir rencontré personnellement je connaissais déjà son visage. Son nom était associé au traitement des irradiés, en particulier à celui des scientifiques yougoslaves. Mais son parcours professionnel touchait en fait à tous les domaines de la radioprotection comme il le soulignait lui-même dans un texte présentant les moments principaux de son parcours professionnel à travers des rubriques aussi diverses que la dosimétrie biologique, la médecine nucléaire, la radiopathologie, la radioécologie, l'hygiène atomique.

Son maître mot fut la multidisciplinarité.

"La découverte des échanges réciproquement enrichissants entre disciplines fondamentales devait marquer de façon définitive ma vie professionnelle ", écrivait-il.

Ce mot signifiait qu'il faisait sentir à chacun, fut-il médecin, ingénieur, biologiste, sociologue, vétérinaire, économiste..., que par sa compétence il était indispensable au développement de la radioprotection. C'est probablement la raison pour laquelle il se sentait bien au sein de la Commission internationale de protection radiologique : lieu de rencontre et de dialogue souvent musclé entre des experts venus de disciplines et de pays différents, cette organisation non-gouvernementale a su à travers plus d'un demi-siècle d'existence conserver tout son poids auprès des autorités réglementaires.

Il y était à son aise car il était aussi et peut être avant tout, un négociateur et un séducteur. Il savait écouter, argumenter, trancher, se mettre en colère 
quand il le fallait, être présent et actif quand les autres se lassaient, ne jamais quitter un lieu de débat trop tôt tant que le texte final n'était pas prêt.

Ce ne fut pas l'un de ses moindres mérites que d'avoir reconnu très tôt l'importance de la confrontation internationale comme source de renouvellement et d'approfondissement de la radioprotection au niveau national.

Lorsque je l'ai rencontré pour la première fois alors que je venais d'être nommée responsable de la radioprotection à l'Institut de protection et de sûreté nucléaire, j'avais beaucoup à apprendre et il savait déjà tout. Il a été un professeur irremplaçable : la rigueur de sa pensée, son esprit de synthèse, son optimisme m'ont profondément marquée et aidée.

A la suite de cette disparition, j'ai reçu de nombreux témoignages dont la finesse d'analyse révèlent la force d'attraction qu'il exerçait sur les autres. Je souhaiterais extraire ces quelques lignes d'une lettre du Dr Burton G. Bennett, Secrétaire de l'UNSCEAR :

"For me, Dr. Jammet represented great strength, stability and understanding that could only be acquired from long years of experience. He was always warm and friendly and more than willing to be helpful and to participate. His long professional career attests to his keen interest in radiation science, his enthusiasm and his desire to serve".

Pionnier, il l'a été aussi par son goût pour le mouvement associatif. Je voudrais rappeler que le Professeur Jammet fut Président de la SFRP de l'année 1966 à la suivante, mais aussi quelques années plus tôt de la Société de radioprotection qui donna naissance par fusion avec la section française de la Health Physics Society, à la SFRP.

Il nous a quittés en plein été. Son amour des voyages l'avait conduit une fois de plus dans un désert qui lui était familier. Mais un pionnier peut-il vraiment disparaître totalement, sauf si l'on décide de ne plus être fidèle à son œuvre? 


\section{hommage à Henri Jammet*}

1920-1996

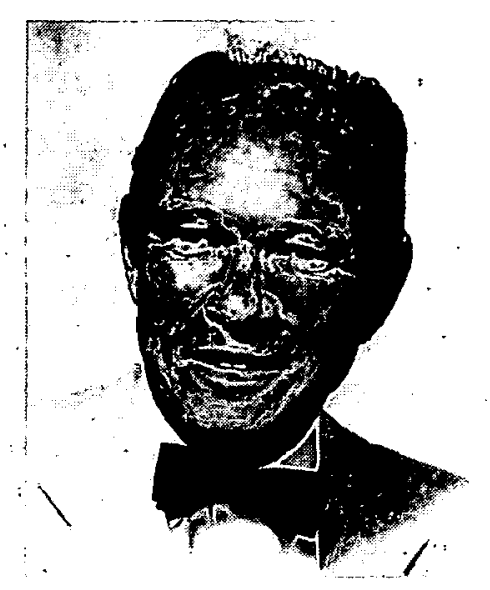

Note du comité de rédaction: Nous avons voulu dans ce numéro rendre un dernier hommage à Henri Jammet. Nous le faisons au travers de ces témoignages reflétant bien toutes ses activités. La qualité de leurs auteurs nous interdit toute hiérarchisation, c'est donc par ordre alphabétique que nous les publions.

H. MÉTIVIER

Henri Jammet was the longest serving Main Commission member, having been continuously in post from 1962 until his death this year. The record of service, some 34 years, is not approached by any other individual. Henri Jammet first joined the ICRP family in 1959 as a member of the then Committee 5 on handling of Radioisotopes and Disposal of Radioactive waste. When the present Committee Structure was introduced in 1962, Henri was elected as Chaiman of the new Committee 4 on the Application of the Commission's Recommandations and thereby joined the Main Commission. His six terms as Chaiman of Committee 4 cannot be matched as he steered that Committee to much of its important work. In 1985 he was elected vice-chaiman of the Commission until in 1993 he took the chair of Committee 3 on Protection in Medicine.

His contributions to radiological protection, both personal and professional, are enormous and his input to the work of ICRP will be sorely missed.

Professor R. Clarke, Chaiman of ICRP

La photo de $\mathrm{H}$. Jammet, publiée dans le $\mathrm{N}^{\circ}$ 3-96 de Radioprotection en page 333, était extraite d'un original en provenance du NRPB, prise lors de l'adoption définitive par la CIPR de ses dernières recommandations. 
Le Professeur H. Jammet était mon ami, depuis le jour où jeune médecin capitaine, je l'assistais très humblement auprès d'un jeune technicien qui, irradié dans un flux neutron-gamma, était arrivé de Mol à l'institut Curie. L'amitié naquit dans l'admiration que le jeune médecin portait au courage, à l'intelligence, à la méthode et à la perspicacité d'un maître.

Au nom du Service de Santé des Armées et dans le groupe du Centre de Recherche de Santé des Armées qui travaillait dans son département au CEA, je fus le témoin de ses qualités et de sa détermination, dans la prise en charge pendant plus de 25 ans de tous les accidents d'irradiés et/ou de contaminés, et dans la direction de son service; il était acquis à la liberté du chercheur et ouvert à tous les axes de la réflexion. Au moment où naissait l'exigence de la radioprotection dans l'ensemble de ses orientations civiles et militaires, il fut un des principaux artisans de sa mise au point et de son évolution dans ses aspects scientifiques et politiques, en France et dans le monde, suscitant parfois jalousie et scepticisme. Il fut présent sur tous les fronts et c'est dans cette perspective qu'il devait créer auprès de l'IRPA le premier comité de radioprotection contre les radiations non-ionisantes; opposant une sérénité de tous les instants aux critiques les plus ignorantes et les plus perverses; il sut dégager en ce domaine l'intérêt de la radioprotection, soutenant notamment l'effort de recherche sur les effets biologiques des lasers.

De plus, loin des aspects techniques, il était un hôte exquis, un compagnon de voyage ou de réunion, dans la réussite comme dans l'infortune, amusant, délicat et attentionné, un polémiste appliqué et toujours respectueux de l'autre, dans des réunions internationales où la politique au prétexte de science adore les attitudes les plus détestables.

Il savait mieux que quiconque, avec V. Jankelevitch, qu'« avec les êtres naturels que nous sommes, il est bon que la loi parle la langue de la nature comme Pascal parle celle des joueurs, tournant l'effort en agrément, l'aversion en attrait puis de nouveau par un aiguillage insensible l'attrait en exigence d'effort ». Il savait aussi mieux que quiconque que «la circonlocution hédonique tourne le devoir en plaisir et retarde, sans l'éluder, le moment de l'effort personnel ». Il en est mort.

Dr Louis Court

Dès les années 50, rapprochant dans ses cours les effets sournois des rayonnements ionisants de ceux d'une exposition prolongée au soleil, le Dr Jammet attirait l'attention sur les effets nocifs immédiats ou à long terme du rayonnement ultraviolet. Par la suite, constatant l'expansion rapide des applications des divers rayonnements non-ionisants (RNI) et l'inquiétude croissante des tra- 
vailleurs et du public quant à leurs effets possibles sur la santé, il fut l'un des premiers à proposer de mener une action internationale afin d'élaborer une politique commune de protection contre les RNI.

C'est pourquoi, en 1974, l'IRPA demanda à H. Jammet d'animer un groupe de travail pour établir un état de la protection contre les RNI à l'échelle internationale, puis le nomma Président du premier Comité international sur les rayonnements non-ionisants (INIRC) créé en 1977 avec mission d'élaborer une doctrine et des limites d'exposition susceptibles d'être adoptées au plan international. Comme Président de l'INIRC de 1977 à 1988, il sut concilier des positions initialement largement divergentes, établir des liens avec d'autres organisations internationales, en particulier l'Organisation Mondiale de la Santé, le Bureau International du Travail et la Commission des Communautés Européennes, et faire du Comité un organisme international dont l'autorité scientifique fut rapidement reconnue dans le monde. Aussi, en signe de reconnaissance, lorsque l'IRPA transforma l'INIRC en une commission indépendante, la Commission internationale de protection contre les rayonnements non-ionisants, ses collègues nommèrent le Dr Jammet Président émérite à vie.

Grâce à l'action menée par le Dr Jammet, la France a pu être présente dans toutes les instances internationales traitant de la protection contre les RNI. En particulier, c'est à la Fondation Curie, sous sa présidence, qu'eut lieu la première réunion d'experts scientifiques pour la préparation de la proposition de directive sur la protection contre les micro-ondes publiée par la CCE en 1980. Il faut également rappeler que c'est à son instigation que la SFRP organisa en 1978, à Nainville les Roches, un congrès international sur les effets biologiques des rayonnements non-ionisants.

A. Duchène

L'une des principales contributions d'Henri Jammet à la radioprotection restera sans conteste sa participation à l'élaboration et la diffusion du principe ALARA qui constitue désormais la pierre angulaire du système de protection radiologique. En tant que Président du Comité 4 de la Commission internationale de protection radiologique (CIPR), qu'il présida sans interruption de 1962 à 1985, il œuvra pour donner à ce principe une consistance méthodologique mais aussi opérationnelle.

L'émergence du principe ALARA a marqué l'ouverture de la radioprotection aux dimensions économiques et sociales et c'est dans cette perspective qu'Henri Jammet décida la création du Centre d'étude sur l'évaluation de la protection dans le domaine nucléaire (CEPN) il y a tout juste 20 ans. Pendant de nombreuses années il en fut l'inspirateur, d'abord comme administrateur, puis comme Président de son Conseil scientifique. C'est dans le cadre des activités du Centre que j'ai eu le privilège de collaborer avec lui. 
Henri Jammet était profondément persuadé que la radioprotection n'est pas seulement une affaire de sciences et de techniques mais aussi une affaire d'économie, de sociologie, de politique et surtout de morale. Il était à la recherche de ce subtil équilibre entre ces diverses dimensions qui, pensait-il, était à même de créer les conditions d'une confiance durable dans la radioprotection et dans les hommes qui la font. Sa parfaite connaissance des fondements biologiques et épidémiologiques sur lesquels reposent la radioprotection moderne le conduisait en permanence à prôner la prudence et le pragmatisme. Il était convaincu qu'éviter toutes les expositions inutiles et maintenir les niveaux d'exposition aussi bas qu'il est raisonnablement possible était la seule attitude vraiment responsable.

Ces dernières années, tout en poursuivant bon nombre de ses activités sur le plan national et international, Henri Jammet s'était engagé dans une réflexion sur l'articulation de la protection avec le droit et l'éthique. Il cherchait à ouvrir une voie pour la gestion des risques. qu'il nommait incertains, pressentant que ces derniers tiendraient tôt ou tard le devant de la scène en matière de gestion des risques.

Mais au-delà du professionnel, c'est l'homme de culture, à la fois grand narrateur mais aussi assez' secret, fasciné par les déserts et les confins des continents, qui restera d'abord dans notre mémoire.

Jacques' Lochard Directeur du CEPN

In 1974, the International Radiation Protection Association (IRPA), became aware of the rapidly expanding uses of non-ionizing radiation (NIR) sources and the health concerns they raised. IRPA asked Henri Jammet to chair a Working group to review the situation concerning occupational and general public protection against NIR, and, in 1977, appointed him as Chaiman of the first International Non-Ionizing Radiation Committee (IRPA/INIRC). This Committee was given responsibility for developing accepted recommendations on protection measures, including exposure limits, for the different NIR. As Chaiman of the IRPA/INIRC from 1977 to 1.988, he set the highest scientific standards for it and its successor, the International Commission on Non-Ionizing Radiation Protection.(ICNIRP). His appointment as the first Chaiman Emeritus of INIRC was testimony, not only to his scientific knowledge in radiation protection, but also to his leadership, his integrity and his personal qualities. 
Avant de jouer un rôle fondamental dans l'évolution de la Radioprotection en France, Henri Jammet avait créé à la Fondation Curie l'un des premiers services de Médecine Nucléaire en France.

J'ai eu la chance d'y travailler comme jeune interne pendant toute une année, ce qui m'a permis d'utiliser le premier scintigraphe fabriqué en France, selon les spécifications d'Henri Jammet.

L'énorme recrutement du service préfigurait les grands services de médecine nucléaire modernes et en particulier ceux des centres anti-cancéreux.

La vivacité et l'extrême clarté dans les décisions du Docteur Jammet animaient l'ensemble et créaient ce ferment d'enseignement qu'il a toujours su entretenir en médecine nucléaire comme en radioprotection.

Pr Claude Parmentier

Henri Jammet est avant tout un grand médecin qui a consacré sa vie à la radiopathologie:

Par sa compétence, son intuition et son sens de la décision médicale, fondés à la fois sur une brillante intelligence, une expérience exceptionnelle, unique en radiopathologie, et un bon sens inébranlable dans la détermination immédiate de l'essentiel, il a acquis, au plan international, la légitime réputation de meilleur spécialiste du traitement des lésions radiologiques les plus graves.

Bien au-delà de la médecine, le champ de ses connaissances s'étendait de la radiobiologie à la physique nucléaire en passant par une solide culture mathématique.

C'est en 1958 à l'Institut du radium, au sein duquel il avait créé le service de radiopathologie selon les dernières volontés d'Irène Curie, qu'il conçut et pratiqua lui-même les premières greffes humaines de moelle osseuse jamais tentées au monde, à la suite de l'accident de criticité nucléaire de Vinca en Yougoslavie.

Ainsi et, ne l'oublions pas, grâce aussi au soutien éclairé, toujours discret et affectueux de Jeanne Jammet, il sauva la vie de cinq des six techniciens irradiés à plus de 600 rads (6 Gy), ouvrant en même temps la voie à une thérapeutique nouvelle qui allait par la suite largement dépasser le domaine de la seule radiopathologie. En cela il fit non seulement œuvre de pionnier, mais encore preuve d'un courage qui est le trait fondamental des hommes de caractère, celui de savoir prendre, dans la solitude, l'entière responsabilité des décisions les plus graves. 
Ce courage, Henri Jammet l'a manifesté en bien d'autres circonstances, aussi bien à l'Institut Curie que comme consultant demandé par les plus grands pays tant sa réputation dépassait nos frontières, lors des divers autres accidents d'expositions lourdes aux rayonnements survenus dans le monde, ceux de Mol, Montpellier, Saintes, mais aussi de Casablanca, Alger, Pékin, Moscou, Minsk, pour ne citer que quelques-uns des plus connus.

Les spécialistes du monde entier lui ont fait confiance, comme en témoigne maintenant leur reconnaissance et leur touchante fidélité, et c'est grâce à sa compétence, son engagement personnel, son légendaire dévouement aux patients, que les conséquences humaines de ces accidents ont pu être limitées à l'extrême.

Sur le plan international, Henri Jammet a toujours été la référence écoutée en pathologie des radiations, ajoutant à son autorité beaucoup de diplomatie, de persuasion, et les qualités pédagogiques que l'on retrouvait dans l'enseignement qu'il dispensait en tant que professeur à l'Institut national des sciences et techniques nucléaires.

Il fut notamment Vice-Président de la Commission internationale de protection radiologique, Président du Centre international de radiopathologie. Peu avant de nous quitter, il venait de créer et présidait l'Association internationale de radiopathologie dans le but d'en fédérer les spécialistes au plan mondial.

Quarante années durant, il aura farouchement défendu le caractère médical intangible de la responsabilité de la radioprotection, et s'il nous arrivait par malheur d'oublier sa leçon et cet impératif, nous prendrions à terme le risque de laisser compromettre la santé de nos concitoyens, sans même évoquer la mise à mal de l'une de nos principalès ressources économiques.

Henri Jammet était du petit nombre de ceux qui ont vécu et connaissent la véritable histoire de notre énergie atomique. C'était un patriote intransigeant. Les services qu'il a rendus au pays sont éminents.

Certes, cet homme hors du commun ne se livrait pas facilement. Persévérant et combatif, mais secret, il ne parlait presque jamais de ses luttes. C'est pourquoi beaucoup ignorent sa véritable personnalité.

Pourtant ceux qui, au-delà des préoccupations professionnelles, ont eu la chance d'apprendre à le connaître, ne sont pas près d'oublier son attachante amitié, son humanisme, son irrésistible humour, l'exceptionnelle étendue de sa culture et de sa mémoire. Il ne laissait personne indifférent.

Reste maintenant un grand vide qu'il ne sera pas facile de combler.

Pierre Pellerin Fondateur du SCPRI 
Henri Jammet a été l'un de ceux qui ont inspiré la radioprotection au cours de la deuxième moitié de ce siècle. Il avait été formé comme radiothérapeute à l'Institut Curie où il avait été d'abord l'élève de François Baclesse. Ensuite, dans les années 50, il s'était tourné vers la médecine nucléaire, discipline jeune et en plein essor. Ceci l'avait mis en relation avec le Professeur Louis Bugnard, le puissant directeur de l'Institut national d'hygiène (qui deviendra, plus tard, l'Inserm). Bugnard s'intéressait beaucoup à la radioprotection mais il le voyait en biophysicien, en théoricien. Il avait besoin, auprès de lui, d'un homme connaissant intimement l'effet des rayonnements sur l'organisme humain, d'un homme de terrain éloigné de tout dogmatisme et de toutes idées préconçues. Il demanda à Henri Jammet de l'aider, et très vite celui-ci s'imposa non seulement par ses connaissances scientifiques et cliniques mais aussi par son réalisme, ses talents de diplomate soucieux d'éviter les conflits tout en améliorant constamment la protection des travailleurs et des malades. En radiobiologiste, il échappait aux terreurs mythiques qu'inspiraient à certains théoriciens les faibles doses des rayonnements et il n'était pas dupe d'un formalisme mathématique qui ne sert qu'à masquer les ignorances. Il aimait la recherche des consensus et il y excellait. Avec autorité, fermeté et souplesse, il mena, en France et sur le plan international, une politique raisonnable de radioprotection. Il tenta de faire entendre la voix de la sagesse face aux concepts dogmatiques et ne perdit jamais espoir de faire prévaloir la raison.

Parallèlement, il mena une grande œuvre de radiopathologiste. L'admirable courage avec lequel il accepta de traiter les cinq victimes yougoslaves d'un accident du réacteur expérimental de Vinca, la ténacité, le sens clinique dont il fit preuve à cette occasion, la façon dont il groupa autour de lui les meilleurs spécialistes français, le plaça au premier plan mondial dans cette discipline dès 1960. Il fut une référence internationale dans ce centre international de radiopathologie, où il recevait de grands irradiés de tous les continents.

M. Tubiana

Membre de l'Académie des Sciences Membre de l'Académie de Médecine 